\title{
Effects of waterbirths and traditional bedbirths on outcomes for neonates
}

\author{
HB Ros, M. (Cur) \\ School of Nursing, University of Johannesburg.
}

\section{Key words}

Waterbirth, traditional bedbirth, neonate, midwife, midwife obstetric unit.

\begin{abstract}
Curationis 32 (2): 46-52
When women are pregnant, some plan to have waterbirths and other plan to have traditional bedbirths. Therefore some neonates will be born under water and other neonates out of the water on a bed. It is unclear what the outcomes for the neonates are after these two types of deliveries. The research goals of this study were to explore and describe the outcomes for neonates after waterbirths (group A) and traditional bedbirths (group B) and to generate hypotheses based on the outcomes for neonates after waterbirths and traditional bedbirths that need to be tested in subsequent research studies. The design was an explorative descriptive survey The mothers were purposefully selected to participate in the research study. They had to be healthy, low-risk pregnant women with a single pregnancy and a cephalic presentation. They had to be 37 to 42 weeks pregnant. Group A delivered their neonates at two private hospitals in Gauteng and group B delivered their neonates at a government hospital in Gauteng. Data was collected during labour, just after the delivery, two hours after the delivery and 14 days after the delivery. A data collection instrument was used. The following neonatal outcomes were measured: Apgar score at one and five minutes, axillary temperature, $\mathrm{pH}$-, haemoglobin- and sodium levels of the umbilical cord blood, the neurological condition of the neonate as reflected by the primitive reflexes and neonatal morbidity until 14 days after birth. Descriptive analysis was used to analyse the data. It appeared if group A had higher Apgar scores, neonatal temperatures and umbilical cord blood haemoglobin levels and lower sodium umbilical cord blood levels than group $\mathrm{B}$. The umbilical cord blood pH levels, neonatal morbidity and primitive reflexes of both groups appeared equal. Group B needed more resuscitation of the neonate directly after birth than group A. Hypotheses were generated that need to be tested in subsequent research.
\end{abstract}

\section{Introduction}

There are many different ways in which pregnant women can deliver their babies. Nowadays, women who deliver their babies vaginally give birth in a hospital setting, lying on a bed - not, any more, at home, squatting or standing on all fours as happened decades ago (Cronjé \& Grobler, 2003: 93). Some women choose to have waterbirths. Waterbirths are increasing worldwide, especially in countries such as South Africa, The USA, Argentina, Belgium, Canada, Chile, China, England, France, Germany, India, Ireland, Israel, Italy,
Mexico, the Netherlands, Russia and Switzerland (Thoni \& Moroder, 2004: 44; Garland, 1996: 27; Shymanski, 1996:1; Alderdice \& Renfrew, 1995: 837; and Geissbuehler, n.d.:1).

Therefore, depending on the birth method chosen by the mother, some neonates are born traditionally on a bed, and others are born under water in a birth tub. It is unclear what the outcomes are for these neonates after each of these two types of delivery.

At birth, fetal neonatal transition takes 
place, which enables the neonate to breathe. In the fetal circulation system there is almost no blood-flow through the liver and the lungs. After birth, the umbilical cord stops pulsating and the foramen ovale, ductus arteriosus and ductus venosus close, causing an increase in the pulmonary circulation as well as in the circulation through the liver (Harrison, 2002: 191). Is there a difference in this process between neonates born under water and neonates born out of the water, traditionally on a bed? How does the type of delivery influence the neonatal Apgar score, temperature, neurological condition and umbilical cord blood pH-, haemoglobin- and sodium levels?

During normal labour there is usually a progressive but small decrease in fetal arterial oxygen tension, oxygen saturation and $\mathrm{pH}$, with a rise in carbon dioxide tension. This is called the transitory asphyxia of the fetus and newborn (Harrison, 2002:162; and Ladewig, London and Olds, 1998:501). These changes probably stimulate the aortic and carotid chemoreceptors that trigger the medulla's respiratory centre to initiate the deep breath that occurs at birth. This impairment is usually corrected within 30 minutes to two hours of age (Harrison, 2002: 162; Mercer and Skovgaard, 2002: 57; Adamson, 1991: 45; and Ladewig et al., 1998: 501).

Harper (2000:1), on the other hand, argues that the acute hypoxia that neonates experience during birth causes apnoea and swallowing, not breathing or gasping. Harper (2000:1) furthermore emphasizes an important point, namely that severe and prolonged lack of oxygen, which occurs when there is fetal distress, may cause the neonate to gasp as soon as it is born, possibly inhaling water into the lungs.

Furthermore, the dive reflex inhibits breathing and can prevent the neonate from breathing. Harper $(2000: 1)$ and Johnson in Garland (1996: 65) explain that the tongue is lined with chemoreceptors or taste buds. When a solution hits the back of the throat, passing the tongue, the taste buds interpret what substance it is, the glottis automatically closes and the solution is then swallowed, not inhaled. The newborn can detect what substance is in his or her throat and thus knows when to swallow and when to breathe. With severe intrapartum hypoxia, though, this mechanism is overridden and the neonate will gasp for air and consequently inhale water when it is born under water.

There are more potential explanations for the initiation of the neonate's breathing after birth, namely touch, cold and sensory stimulation (Mercer and Skovgaard, 2002: 57; and Adamson, 1991: 45). Thus, the neonate will not start to breathe after birth when he or she has not been stimulated or exposed to cold.

During waterbirth there is a reduction of stimulation to the neonate. The baby is born in warm water. The temperature of the water is so close to that of the maternal temperature that it prevents any detection of change by the newborn, thus preventing the initiation of breathing (Harper, 2000: 1).

A final inhibitory factor is the water itself. Water is a hypotonic solution, and the lung fluids present in the fetus are hypertonic. So, even if water was to enter past the tongue, it could not pass into the lungs, since hypertonic solutions are denser than hypotonic solutions, preventing the hypotonic solutions from entering the lungs (Harper, 2000:2).

It is unclear what the Apgar scores are of the neonates born under water and traditionally. Garland (2002: 508) and Geissbühler and Eberhard (2000: 296) argue that Apgar scores after waterbirths are higher than after bedbirths, but Rodrigo, Brown, Williams and Cartmill (n.d.: 108) and Nikodem (1999: 230) find no statistically significant difference in Apgar scores between neonates delivered under water and traditionally.

Although a neonate has high Apgar scores at birth, the neonate may still have a poor condition at birth owing to severe hypoxia in utero, and so may have cord blood acidaemia. The $\mathrm{pH}$ level is thus a more accurate reflection of the neonate's condition at birth (Harrison, 2002: 165). Some authors find the cord blood $\mathrm{pH}$ of babies after waterbirth higher than after traditional bedbirths while other authors find no significant difference (Schrocksnadel, Kuncziky, Meier, Brezinka \& Obcraigner, 2003: 7; Geissbühler and Eberhard, 2000: 296; and Nikodem, 1999: 232).

Hyperthermia caused by prolonged exposure to too warm water is associated with hypoxic ischemic encephalopathy of the neonate (Pinette et al., 2004: 1214; and Rosevear, Fox, Marlow \& Stirrat, 1993:342). It is advised that the temperature of the water must be kept between $36^{\circ} \mathrm{C}$ to $37^{\circ} \mathrm{C}$ (Odent, 2004: 107). It is unknown whether the above mentioned water temperature will cause normal neonatal temperatures in neonates born under water.

It is unclear what the neurological condition of the neonates at birth, as reflected by primitive reflexes, is of neonates born under water and those born traditionally. It is, moreover, not known whether the waterbirth process, which includes warm water immersion and the neonate that is born under water, has an influence on the neonatal cord blood values.

Infective conditions in neonates born under water have been reported by Rawal, Shah, Stirk and Mehtar (1994: 511), Pinette et al. (2004:1214), Bowden, Kessler, Pinette and Wilson (2003: 973), Parker and Bowles (1997: 653) and Kingsley, Hutter, Green and Speirs (1999: 155). Therefore the impact and influence of waterbirth on neonatal infections must be investigated. An effective water birthing infection control protocol should be followed to avoid neonatal infections (Kingsley et al., 1999: 155; and Roome \& Spencer, 1996: 274). What is the infection rate in neonates born under water and traditionally?

\section{Problem statement}

Although water births are currently receiving attention, in South Africa there is dearth of literature related to this delivery. It is therefore important to conduct research in this area. In a normal traditional birth, a neonate is stimulated to breathe after birth due to coldness. Mercer and Skovgaard, (2002:57) and Adamson (1991:45) found out that neonates give their first breaths as soon as they are exposed to cold air after birth. In contrast to this, when neonates are born in warm water, they 
are not exposed to coldness and therefore the neonates' first breaths are delayed. Furthermore, the dive reflex causes the neonates to swallow and not to breathe under water (Harper, 2000:1; and Johnson in Garland, 1996:65). The researcher therefore would want to find out if swallowing of water influences the neonates' condition at birth.

Evidence of the effect of waterbirth and traditional bedbirth on the outcomes for neonates, as far as respiration, thermoregulation, adaptation to the environment, neurological condition and general well-being are concerned, is inconsistent and controversial. Little research has been conducted in this field in South Africa, despite the increased demand by consumers that makes such research necessary. The researcher is interested in answering the following questions:

- What are the outcomes for neonates after waterbirths and traditional bedbirths?

- Which hypotheses on the outcomes for neonates after waterbirths and traditional bedbirths can be generated that need to be tested in subsequent research studies?

\section{Research objectives}

The research objectives of this study were:

- To assess the outcomes for neonates after waterbirth and traditional bedbirth as reflected in the following specific measurable criteria:

- Apgar score at one and five minutes,

- temperature of the neonate as soon as possible after birth,

- umbilical cord blood $\mathrm{pH}$,

- umbilical cord blood haemoglobin and

- umbilical cord blood sodium;

- To explore and describe the neurological outcomes for the neonates by assessing the primitive reflexes approximately two hours after birth;

- To explore and describe neonatal morbidity 14 days after delivery; and

- To generate hypotheses on the outcomes for neonates after waterbirth and traditional bedbirth that should be tested in subsequent research studies.

\section{Definitions}

Water birth is when the mother, who is in a birthing tub filled with warm water during labour, gives birth to her neonate under water in the birthing tub (Scott, 2001:13). The water is deep enough to support the mother so that she can derive comfort from its buoyancy, but should not be so deep that she feels insecure (Scott, 2001: 6; and Lichy \& Herzberg, 1993: 102).

The neonate is delivered completely under the water and is then taken to the surface slowly and gently after birth, the birth attendant lifting the neonate's head first out of the water. Once the neonate's head is lifted out of the water, it is not submerged again (Geissbühler \& Eberhard, 2000: 292; Harper, 2000: 3; and Nikodem, 1999: 16). The waterbirths included in this study occurred in active birth units in private hospitals. The birth attendants were midwives.

Traditional bedbirth is when the delivery is managed with the mother in a semi-Fowlers or lithotomy position, lying on a bed. This is the common practice in hospital settings nowadays (Cronjé \& Grobler, 2003: 79). In this study, traditional bedbirths are taken to mean births of this kind in a labour ward in a governmental hospital, where no epidural anaesthesia is given to the mother. The birth attendant is a midwife.

A neonate refers to a baby from birth until 28 days of age (Harrison, 2002: 1; and Pieterse, 1993: 383).

A midwife is a person who has successfully completed the prescribed course of studies in midwifery and has acquired the requisite qualifications to be registered and/or legally licensed to practise midwifery. She is able to give the necessary supervision, care and advice to women during pregnancy, labour and the postpartum period, to conduct deliveries on her own responsibility and to care for the newborn and the infant (Nolte, 1998: 3).

A midwife obstetric unit is a unit en- tirely staffed by midwives, who assume primary responsibility for ante-, intraand postpartum carc. Medical care is easily available. The midwife obstetric unit deals with normal low-risk mothers, but those with complications are referred (Nolte, 1998: 6). In this study, all the women delivered their neonates in a midwife obstetric unit. The midwife obstetric unit where group $A$ (waterbirth) delivered their neonates, was at two active birth units in private hospitals and the midwife obstetric unit where group B (traditional bedbirth) delivered their neonates was in a labour ward at a government hospital.

\section{Research methodology Research design}

An explorative descriptive survey was done to explore and describe the effects of waterbirth and traditional bedbirth on the outcomes for neonates. Hypotheses were generated based on the results. The neonate was the unit of analysis (Babbie \& Mouton, 2001: 232).

\section{Population and sampling}

The population include all the neonates of mothers that delivered in a labour ward at a government hospital and two active birth units at private hospitals in Gauteng. The sample was taken purposively from the population: the pregnant mothers who met the inclusion and exclusion criteria, as described in the following two paragraphs, were asked to participate in the research study and those who agreed were included in the research study. The sample consisted of two groups: group A, where all the mothers had waterbirths in active birth units at private hospitals and group B, where all the mothers had traditional bedbirths in a labour ward at a government hospital in Gauteng.

\section{Inclusion criteria}

The inclusion criteria involve a list of characteristics essential for membership in the population from which the sample was selected. The inclusion criteria were designed to make the population and sample as homogeneous as possible (Burns \& Grove, 1993: 236). The inclusion criteria that were set are as follows: the mothers had to be healthy, low-risk pregnant women with a single pregnancy and a cephalic pres- 
entation. The gestational age on the day that the neonate would be born had to be 37 to 42 weeks.

\section{Exclusion criteria}

A list of exclusion criteria was set to limit the effect of extraneous variables on the particular interaction between the dependant and independent variables (Burns \& Grove, 1993: 403). If the mothers had one or more of those conditions listed in the exclusion criteria list, they were excluded from this research study. The exclusion criteria list that was used in this research study was as follows: anaemia (where the haemoglobin level is less than $10 \mathrm{~g} / \mathrm{dl}$ ), cardiac disease, lung disease, renal disease, diabetes, epilepsy, rhesus sensitisation, pre-eclampsia, eclampsia, hypertension, thick meconium stained amniotic fluid, rupture of membranes for longer than 24 hours, excessive vaginal bleeding, severe impaired fetal growth, preterm labour, HIV, hepatitis and herpes infection, amnionitis, previous Cacsarean section, any presentation which is not cephalic, and a multiple pregnancy.

\section{Data collection}

Data was collected by the researcher and the attending midwife during labour, just after the delivery, two hours after the delivery and 14 days after the delivery. The researcher was responsible for recording all the data obtained in the data collection instrument.

The mother's biographical information was obtained and screened to establish whether she met the inclusion and exclusion criteria. The fetal condition during first stage and second stage was recorded, as well as the type of pain relief the mother received during labour. The temperature of the mother, the water in the birth tub and the neonate, as well as the Apgar scores, were determined and recorded. Umbilical cord blood was obtained and sent to the laboratory for the determination of the $\mathrm{pH}-$, haemoglobin- and sodium levels. Any complication that occurred and any resuscitation that was needed were recorded. The neonate's primitive reflexes were tested approximately two hours after birth. The mother's file was audited to gain additional information regarding the labour and the delivery process and neonatal details. The mother was phoned fourteen days after the delivery to enquire about her baby's health.

\section{Validity and reliability}

Internal validity was ensured by selecting the sample based on an inclusion and exclusion criteria, by using the same data collection instrument for both groups which was based on national and international scientific literature and which was discussed and validated by both the researcher and the study leader. A statistician of the University of Johannesburg was consulted about the instrument which was adapted to make data capturing and analysis easier with the use of a computer. All the participating midwives were trained by the researcher on the research protocol that was followed. This ensured consistency of the evaluations to have valid results and to minimise the influence of extraneous variables. The research protocol was based on an extensive literature survey of national and international studies, and validated by two midwives experienced in both methods of delivery. The same type of thermometer was used every time by the researcher.

Questionnaire validity was ensured by the fact that the same person, namely the researcher, did the telephonic interview 14 days after delivery, with the mothers.

External validity was ensured by consulting a statistician to choose a suitable sample size. The study sample is representative of the healthy pregnant women that attend a labour ward at a government hospital and two active birth units at private hospitals in Gauteng.

Reliability was ensured by using the same equipment, questionnaire and research protocol for all the women included in the study. The equipment used was fully functional, which ensured measurement reliability. Noncomparable results were avoided by training all the midwives in the research protocol and by sending the umbilical cord blood to the same laboratory for analysis.

\section{Ethical issues}

- Clearance of the ethical committee at the University of Johan- nesburg was obtained.

- Permission to conduct the research study was gained from the various hospitals' nursing management, the attending midwives and the participants. They gave informed consent

- The participants were reassured that their names and information would be held private and confidential and that no pain or harm would be inflicted to them or their babies. It was explained to them that their participation would be voluntary and that they could withdraw from the study at any time if they wished to do so.

- The patients, midwives and hospitals' nursing management were reassured that the research study had no financial implications for them.

- The researcher respected the professionalism of the attending midwives and did not interfere with the nursing care, management and treatment of both the mothers and the neonates.

\section{Data analysis}

Descriptive analysis was used to explore and describe the outcomes for the neonates after waterbirths and traditional bedbirths. This was a method for presenting quantitative description in a manageable form (Babbie $\&$ Mouton, 2001:459). The umbilical cord blood was sent to the same laboratory for the determination of the $\mathrm{pH}-$, haemoglobin- and sodium levels.

\section{Results of the data analysis}

The following outcomes for the neonates were analysed: Apgar score at one and five minutes, temperature of the neonate as soon as possible after birth, umbilical cord blood $\mathrm{pH}$-, haemoglobin- and sodium levels, primitive reflexes and neonatal morbidity. See table I for the values of the above mentioned neonatal outcomes of both groups: group A, the waterbirth group and group $B$, the traditional bedbirth group.

Group B (traditional bedbirth) had lower Apgar scores than group A (waterbirth). The marker for a low Apgar score is an Apgar score of less than 


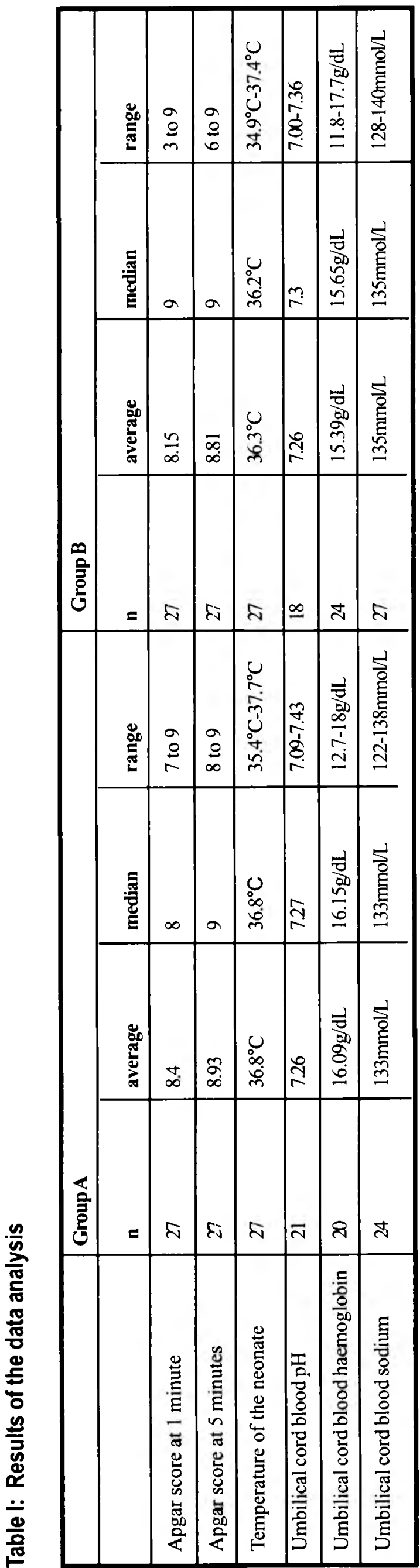

seven (Garland, 2002: 510).

Eleven percent $(3 / 27)$ of the

Apgar scores at one minute and $4 \%(1 / 27)$ of the Apgar scores at five minutes of group B (traditional bedbirth) were less than seven. None $(0 \%)$ of the Apgar scores of group A (waterbirth) were less than seven.

The neonatal temperatures of group B (traditional bedbirth) were also lower than the neonatal temperatures of group A (waterbirth). Forty one percent (1 1/27) neonates of group B (traditional bedbirth) versus $15 \%(4 / 27)$ neonates of group A (waterbirth) had hypothermia (an axillary temperature of less than $36.2^{\circ} \mathrm{C}$ ). Group A (waterbirth) had 4\% (1/27) neonates with hyperthermia (an axillary temperature of greater than $37.5^{\circ} \mathrm{C}$ ) versus group $\mathrm{B}$ (traditional bedbirth), who had none $(0 \%)$.

The average umbilical cord blood $\mathrm{pH}$ of both groups were equal, namely 7.26. This indicated neonatal acidosis, since the normal range of the $\mathrm{pH}$ level for a neonate on the first day of his/her life is 7.27-7.47 (Ampath, 2005: 1). Harrison (2002: 162) explains that during normal labour there usually is a small but progressive decrease in the $\mathrm{pH}$ level. Therefore a $\mathrm{pH}$ of 7.26 , which indicates slight acidosis, can be seen as normal in a neonate a few minutes after birth. Group A (waterbirth) had a slightly higher umbilical cord blood haemoglobin and a slightly lower umbilical cord blood sodium level than group B ( traditional bedbirth). The umbilical cord blood haemoglobin and sodium levels were within normal limits.

Four primitive reflexes of the neonate were tested approximately two hours after birth, namely the palmar grasp reflex, the plantar grasp reflex, the Moro reflex and the rooting reflex. The neonate scored two points when the reflex was present, one point when the reflex was slow and zero points when the reflex was absent. All these points of the four reflexes were added up to give a total out of eight for each neonate. All the neonates' totals were added up to give a total out of 54 for each group. The total score of primitive reflexes of neonates for each group is presented in Table II. The neonates had good palmar grasp, plantar grasp and Moro reflexes, but no good rooting reflexes. The reason why the rooting reflexes were absent is not clear.

Just after delivery, the following resuscitation on the neonates were needed:

from group A (waterbirth):

- five neonates were suctioned through their mouths and noses;

- three received oxygen through a funnel;

- two received Narcan intra-muscularly;

- four of the neonates' mothers were given Narcan intraveneously just before delivery; and

from group B (traditional bedbirth):

- $\quad$ six neonates were suctioned through their mouths and noses;

- $\quad$ one neonate was bagged with oxygen and received oxygen through a funnel a few minutes later;

- four more received oxygen through a funnel;

- four received Narcan intramuscularly; and

- $\quad$ one neonate's mother was given Narcan intravenously just before delivery.

It looked like the neonates of group A (waterbirth) needed less resuscitation than the neonates of group B (traditional bedbirth).

None of the neonates of either group was admitted to the neonatal intensive care unit immediately after delivery. One neonate of group A (waterbirth) was diagnosed with sepsis and congenital pneumonia ten hours after delivery and was then admitted to the neonatal intensive care unit. Investigations were done, but the cause for 


\begin{tabular}{|l|l|l|l|l|}
\hline & Palmar grasp reflex & Plantar grasp reflex & Moro reflex & Rooting reflex \\
\hline A (waterbirth) & $54 / 54$ & $51 / 54$ & $54 / 54$ & $26 / 54$ \\
\hline B (traditional bedbirth) & $54 / 54$ & $52 / 54$ & $52 / 54$ & $36 / 54$ \\
\hline
\end{tabular}

the infection in the neonate remained unknown.

All the mothers replied during the telephonic interview 14 days after the birth of their babies, that their babies were healthy. One mother in each group could not be contacted.

\section{Limitations of the study}

The sample size was small: there were 27 participants in each group. This decreased the generalisation to a bigger population and the sample size was too small to make comparisons between the two groups (Burns \& Grove, 1993: 248)

The umbilical cord was clamped a little bit later in group A (waterbirth) than group B (traditional bedbirth), because a neonate born under water has to be lifted up out of the water and the water in the birth tub has to be drained to lower its level before the umbilical cord can be clamped and cut. The umbilical cord may not be clamped and cut under water. This could have influenced the umbilical cord blood haemoglobin values at birth.

A last limitation was that it was not possible to analyse all the umbilical cord blood of the neonates, owing to the fact that some of the blood clotted, and other reasons. This made the sample size for the umbilical cord blood values even smaller.

\section{Recommendations}

\section{Recommendations for nursing practice}

A specific research protocol was followed during this research study. No adverse effects were seen in the outcomes for neonates delivered by both waterbirth and traditional bedbirth. Therefore it is recommended that the research protocol of this research study should be followed when conducting a waterbirth and/or a traditional bedbirth. It is recommended that the following aspects should be adhered to when conducting waterbirths and/ or traditional bedbirths:

- Clean the birthing tub system thoroughly to prevent infection. Use Biocide D to clean the birth tub before and after each delivery and use Hibiscrub to clean the birth tub just before the woman enters the birth tub.

- $\quad$ Add salt to the water in the birth tub to prevent hyponatremia.

Add $1 \mathrm{~kg}$ of coarse salt to every 100 litres of water. When water will be added to the birth tub at a later stage, add coarse salt related to the amount of water that was added (e.g. $10 \mathrm{~g}$ coarse salt for every 1 litre of water).

- Monitor the fetal heart rate regularly and accurately to ensure that there is no fetal distress when a waterbirth is planned to prevent wateraspiration.

- $\quad$ Keep the temperature of the water in the birth tub between $36^{\circ} \mathrm{C}$ and $37^{\circ} \mathrm{C}$ and use a medical industrially manufactured thermometer to measure the water temperature accurately. This will prevent premature breathing due to too cold water temperatures and neurological damage and/or neonatal death due to prolonged exposure to water temperatures that are too high.

- $\quad$ Keep the room temperature of the delivery suite above $23 \mathrm{U} C$, dry the neonates and keep them warm by promoting skin-to-skin contact between the mothers and their babies to prevent neonatal hypothermia (Harrison, 2002: 25; and Simchak, 2003: 24).

\section{Recommendations for midwifery education}

Add an additional lesson unit about waterbirths to the student nurses' training which contains the following content: exclusion criteria for waterbirth, cleaning of the birth tub, establishing and maintaining of the salt content of the water, the importance and method of monitoring the fetal heart rate, the maternal temperature and the water temperature, the range of the recommended water temperature and how to deliver a neonate under water.

\section{Recommendations for future research}

Future research must test the hypotheses generated in this study (Ros, 2006:65).

\section{Conclusion}

Differences were seen in neonatal outcomes between waterbirths and traditional bedbirths. Based on these results, hypotheses were generated that need to be tested in future research studies. A bigger sample size will be needed to be able to generalise the findings to the population.

\section{Acknowledgements}

Sincere gratitude is acknowledged to Ampath Pathologists for financial assistance.

I wish to express my gratitude for the opportunity that was given to me to present this research study at the $6^{\text {th }}$ Annual Congress of Midwives of South Africa on 6 December 2006, at the Sensitive Midwifery Symposium on 18 May 2007 , at the Nursing Odyssey Congress on 9 July 2007 and at the $24^{\text {th }}$ Quadrennial Congress of the International Council of Nurses on 3 July 2009.

\section{References}

ADAMSON, SL 1991: Regulation of breathing at birth. Journal of Develop- 
mental Physiology. 15( 1 ): 45-52.

ALDERDICE, F \& RENFREW, M 1995: Labour and birth in water in England and Wales. British Medical Journal, 310(6938): 837.

AMPATH 2005: Final Report: Haematology and Biochemistry. Pretoria: Ampath Pathologists.

BABBIE, E \& MOUTON, J 2001: The practice of social research. Cape Town: Oxford University Press Southern Africa.

BARRY, CN 1995: Water births. British Medical Journal. 310(6994): 1602.

BOWDEN, K; KESSLER, D; PINETTE, M \& WILSON, D 2003: Missing the evidence or missing the point? Pediatrics, 112: 972-973.

BURNS, N \& GROVE, K 1993: The Practice of Nursing Research: Conduct, Critique and Utilization. Second cdition. London: Saunders.

CRONJÉ, HS \& GROBLER, CJF 2003: Obstetrics in Southern Africa. Second edition. Pretoria: Van Schaik.

GARI.AND, D 1996: Waterbirth: An Attitude to Care. Cheshire: Books for Midwives Press.

GARLAND, D 2002: Collaborative waterbirth audit-supporting practice with audit. MIDIRS Midwiferv Digest. 12(4): 508-511.

GEISSBUEHLER, V n.d.: Frauenklinik Frauenfeld: waterbirths. Available from: http://www.frauenklinik-frauenfeld.ch (Accessed 9 March 2005).

\section{GEISSBÜHLER, V \& EBERHARD, J} 2000: Waterbirths: a comparative study. Fetal Diagnosis Therapy. 15(5): 291-300.

HARPER, B 2000: Waterbirth basics: from newborn breathing to hospital protocols. Midwifery Today, 54: 1-8.

HARRISON, VC 2002: The Newborn Baby. Cape Town: Mills Litho.

KINGSLEY,A; HUTTER,S; GREEN, N \& SPEIRS, G 1999: Waterbirths: regional audit of infection control prac- tices. Journal of Hospital Infection. 4I: 155-157.

LADEWIG, PW; LONDON, ML \& OLDS, SB 1998: Maternal-newborn Nursing Care. New York: AddisonWesley.

LICHY, R \& HERZBERG, E 1993: The Waterbirth Handbook: the Gentle Art of Waterbirthing. Wellow: Gateway Books.

MERCER, JS \& SKOVGAARD, RL 2002: Neonatal transitional physiology: a new paradigm. Journal of Perinatal \& Neonatal Nursing, 15(4): 56-76.

NIKODEM, VC 1999: The Effects of Water on Birth: A Randomised Controlled Trial. PhD thesis. Johannesburg: Rand Afrikaans University.

NOLTE, AGW 1998: A Textbook for Midwives. Pretoria: J.L. van Schaik.

ODENT, M 2004: The Cacsarean. Sidmouth: Chase Publishing.

PARKER, C \& BOWLES, RG 1997:

Pseudomonas otitis media and bacteremia following a water birth. Pediatrics. 99(4): 653-654.

PIETERSE, H (ed.) 1993: Mediese Woordeboek: Vertalend en Verklarend. Oxford: Oxford University Press.

PINETTE, MG; WAX, J \& WILSON, E 2004: The risks of underwater birth. American Journal of Obstetrics and Gynaecology, 190(5): 1211-1215.

RAWAL, J; SHAH, A; STIRK, F \& MEHTAR, S 1994: Waterbirth and infection in babies. British Journal of Midwifery, 309(8): 511 .

RODRIGO, WN; BROWN, L; WILLIAMS, C \& CARTMILL, RSV n.d.: Delivery under water, safety and how different it is from conventional births. Unknown Journal. P2.02.04.

ROOME, APCH \& SPENCER, RC 1996: Birthing pools and infection control. The Lancet, 348(9022): 274.

ROS, HB 2006: An explorative descriptive survey of the outcomes for neonates after waterbirths and traditional bedbirths. Mini-dissertation.
Johannesburg: University of Johannesburg.

ROSEVEAR,SK; FOX, R; MARLOW, N \& STIRRAT, GM 1993: Birthing pools and the fetus. The Lancet. 342(8878): 1048 .

SCHROCKSNADEL, H; KUNCZICKY, V; MEIER, J; BREZINKA, $C$ \& OBERAIGNER, W 2003: Water birth: experience at a university clinic and a district hospital in Austria. Gynakol Geburtshilfliche Rundsch.43(1): 7-11.

SCOTT, P 2001: Active Birth Pools. Available from: http:// www.activebirthpools.com (Accessed 8 March 2005).

SHYMANSKI, J 1996: Sea of Love: Waterbirth, a Way of Life for Russian Couples. Available from: http:// www.cgi.cnn.com (Accessed 9 March 2005).

SIMCHAK, M 2003: Kangaroo Mother Care. International Journal of Childbirth Education. 18(1):24-25.

THONI, A \& MORODER, L 2004: Waterbirth, a safe and natural delivery method: experience after 1355 waterbirths in Italy. Midwifery Todav. 70:44-48. 